\title{
A Design and Implementation of the Remote Control Black Box System of Vehicle Using the Smart Phone
}

\author{
Jong Geun Song, Won-Tae Jang, and Tae Yong $\mathrm{Kim}^{\dagger}$, Member, KIMICS
}

\begin{abstract}
This paper suggests the vehicle remote control on the basis of Smart Phone. In general, most smart phone is mounted with G-sensor to control the motion. G-sensor is able to control several directions and movements of velocity along with $X, Y$, and $Z$ axis. To access remote location and data system, we can also utilize Wi-Fi communication as well as bluetooth communication. In this study, we propose the scheme that is the car management application by remote control via real-time monitoring on mobile device for user convenience.
\end{abstract}

Index Terms - Mobile Service, Remote Control, Wi-Fi, Vehicle, Network.

\section{INTRODUCTION}

CONTROLLING terminal equipments through network has become considered, and wireless communication technologies and various terminals can be used to access remote location and file system. Especially ubiquitous computing environment is shown as the technology of information communication field including computer, software, and network corresponding to spread much business service [1].

A number of retained domestic vehicles has been increasing continually in the previous 10 years, so much problems such as increasing car accident, a traffic jam, and damaged road are provoked. To avoid the problems, telematics service has received attention as the way to solve those problems. The government also recognizes telematics for the next generation main force IT area and selects it as one of the 9th new development power to push ahead with business activation. Moreover, the black box, which has been used to ascertain the cause when plane accident occurs, is also actively utilized to investigate the cause of car accident.

Utilizing extra camera and vehicle image black box of hardware type such as DVR run to waste the most of the studies on black box for car accident reappearance and there would be additional expense because extra hardware equipments are required to make use of it.

\footnotetext{
Manuscript received October 13, 2010; revised November 16, 2010; accepted November 25, 2010.

${ }^{\dagger}$ Corresponding Author: Tae Yong Kim is with the Div. of Computer and Information Engineering, Dongseo University, Busan, 617-716, Korea (Email: tykimw2k@gdsu.dongseo.ac.kr)
}

Therefore, this paper designates black box system of vehicle remote control based on Smart Phone to handle the problems in accordance with the advantages that cannot add flexible functions to receipt or notify accidents automatically.

It has been generally researched to control in a short range distance with using $\mathrm{RS} 232 \mathrm{C}$ communication method which has been used for the technology of vehicle remote control until today. This method has not been able to investigate directly to the actual vehicle and it is the existing investigation of controlling vehicle by using the models. However, there is some problem for realizing ubiquitous computing environment, since the remote system controlling with wireless LAN (Wi-Fi) should be limited. Also bluetooth technology is only available to control in arranged Wi-Fi service area or within the bluetooth communication distance.

Wi-Fi basically supports super high-speed long distance communication at the same level of cable internet as a wireless internet technology providing stable transmission speed in circumstances of moving with the use of portable terminals; therefore, vehicle control and/or confirmation of vehicle condition will be able to be operated via portable terminal even in an out-of-the-way place by installing the required equipments for image processing of vehicle which demands much calculation [2].

In order to control a vehicle with wireless, the portable iPhone terminal and the iPod touch in which Wi-Fi and bluetooth communication is possible. It is difficult to substitute the real vehicle because the tilt sensor, the ultrasonic sensor, and radio communication camera in the $\mathrm{RC}$ car cannot be easily mounted. Besides that, it is limited to use bluetooth function with iPhone; thus, a system - controlling via mediation PC server to mediate in control information and monitoring information - is established.

The control equipment of vehicle is planned to control with using a mounted G-sensor on the iPhone and iPod touch terminals and it can also view the condition of moving vehicle with image data. Such transmission functions towards terminal have been added through notification message, sound, and vibration for vehicle condition; therefore, more accurate driving decision will be made since those functions help to request information in a real time as well as notify the problems of vehicle condition. 


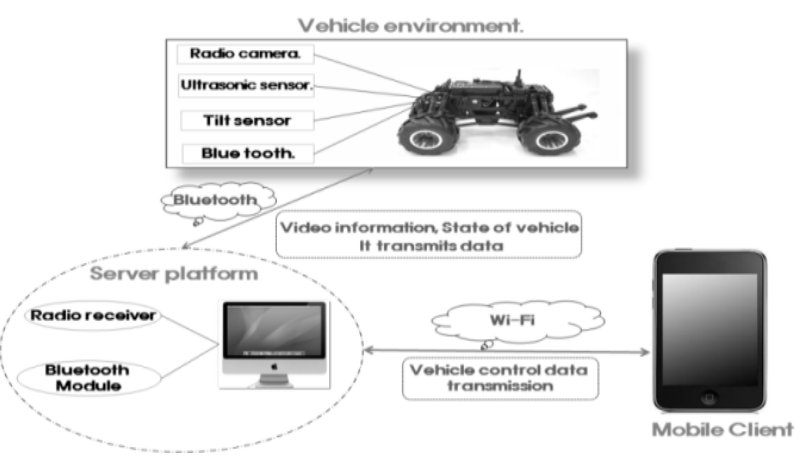

Fig. 1 The configuration for the control system.

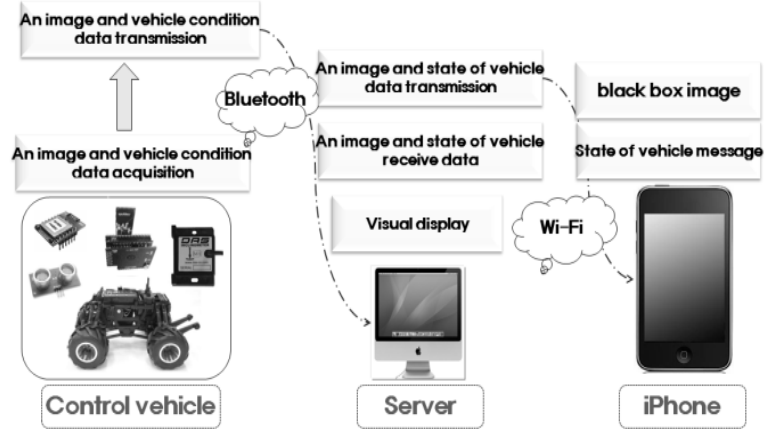

Fig. 2 The communication is performed from remote controlled vehicle to remote terminal.

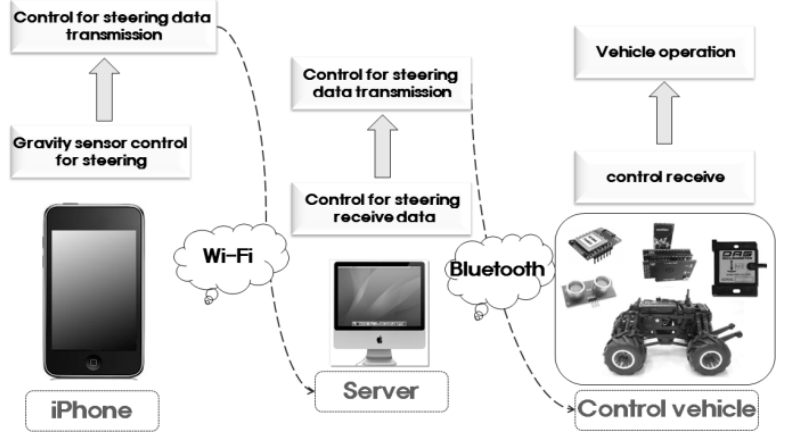

Fig. 3 The communication is performed from iPhone to remote controlled vehicle.

\section{VEHICLE REMOTE CONTROLLING}

\section{A. Vehicle Remote Control}

Vehicle remote control section using $\mathrm{Wi}-\mathrm{Fi}$ and Bluetooth network can be widely divided into controlled vehicle (client), server PC, and remote terminal (client). Fig. 1 presents the whole system for the vehicle remote control designated in this paper. This system is consisted of two large sections. Fig. 2 shows the communication composition from controlled vehicle to remote terminal and Fig. 3 presents the communication is performed from iPhone to remote controlled vehicle.

\section{B. Composition and role of controlled vehicle}

The role of image processing part saves image information of changing front road in a real time through wireless camera installed at the front section of vehicle, and then sends data to server PC by using wireless transmitter. The received data image file from PC Server becomes transmitted to portable terminal through $\mathrm{Wi}-\mathrm{Fi}$ communication following the frame setting per second and this image can be viewed from Server in real time. The size of image data transmitting to terminal via two stages such wireless receiver and Wi-Fi optimizes frame size to procure a stable transmission rate.

The image data obtained from wireless camera is transmitted to iPhone terminal by the way of PC Server through wireless network and remote vehicle can be controlled by monitoring this image data. At this point, a system is built to substitute steering apparatus of vehicle by controlling scope information along with the loaded $G$ sensor to iPhone terminal.

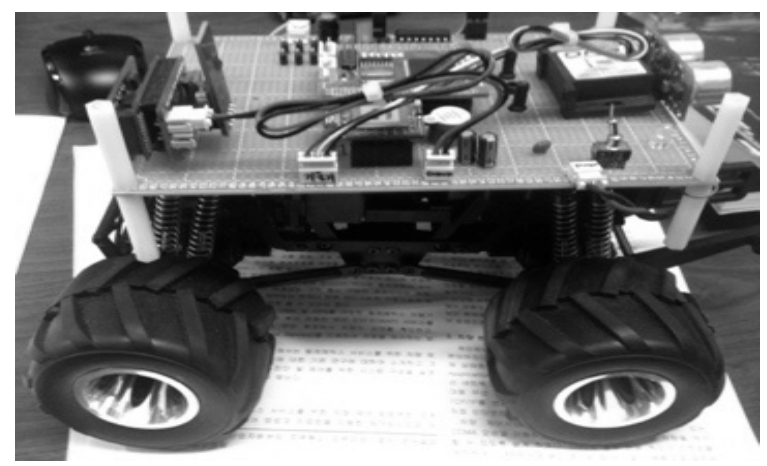

Fig. 4 The vehicle model for remote control system.

\section{Composition and role of remote controlled server}

The remote server part of vehicle remote control equipment is widely divided into image data transmitter/receiver part and vehicle data transmitter/ receiver part.

The transmission part of image data receives data from wireless camera of vehicle by wireless receiver of PC Server and sends image as iPhone terminal through Wi-Fi by changing the received data to image. At this point, image is presented simultaneously with server and terminal in real time.

The transmitter/receiver part of vehicle data is transmitted to server by monitoring the moving condition of vehicle in real time with using the installed slope sensor and ultrasonic sensor, up to this point, communication with server is fulfilled by utilizing Bluetooth SPP (Serial Port Profile) to transmit condition information of vehicle. Furthermore, server transmits the data received from vehicle to the terminal by Wi-Fi so that the vehicle condition can be controlled with monitoring [3]. 


\section{VEHICLE REMOTE CONTROL SYSTEM}

Fig. 5 presents the frame structure for wireless data communication. It divides the type of packet using the first 1-byte information from data frame and the next 1byte is used to have a structure saving the size of packet. From the next bytes, the data actually transmitting is saved to be byte block.

At the early stage of communication, the type of packet and size information are grasped by reading 2-bytes of the frame front part and the next part of data is being read based on the information of packet size afterward. The packet size reading up to the point becomes dependant on the sort of packet type. The kind and use of packet type is same as follows.

Packet Type 1: Packet in which the vehicle steering information of iPhone transmits to remote controlled vehicle iPhone.

Packet Type 2: Packet which transmits the image information obtained from remote controlled vehicle to iPhone.

Packet Type 3: Packet in which the information of ultrasonic sensor as well as slope sensor obtained from remote vehicle transmits to iPhone.

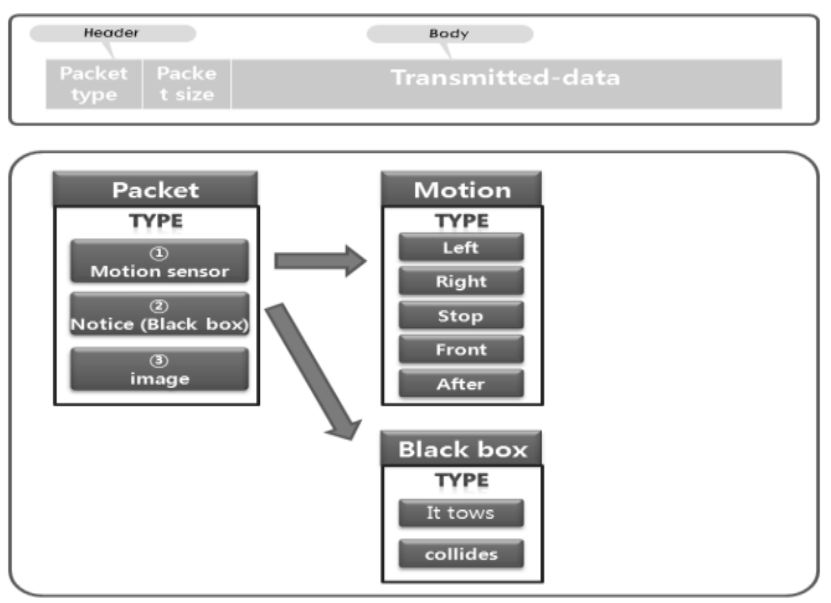

Fig. 5. The caption comes after the figure.

Remote server shown in Fig. 6 is largely formed with packet analyzer, communication module, image module, and client management module. iPhone terminal client or wireless camera fulfills data communication with server by using Bluetooth. Communication data transmits as a stream data format and each packer is handled through packet analyzer. Image management module changes stream data to image data and fulfills such functions to save or manage after compressing.

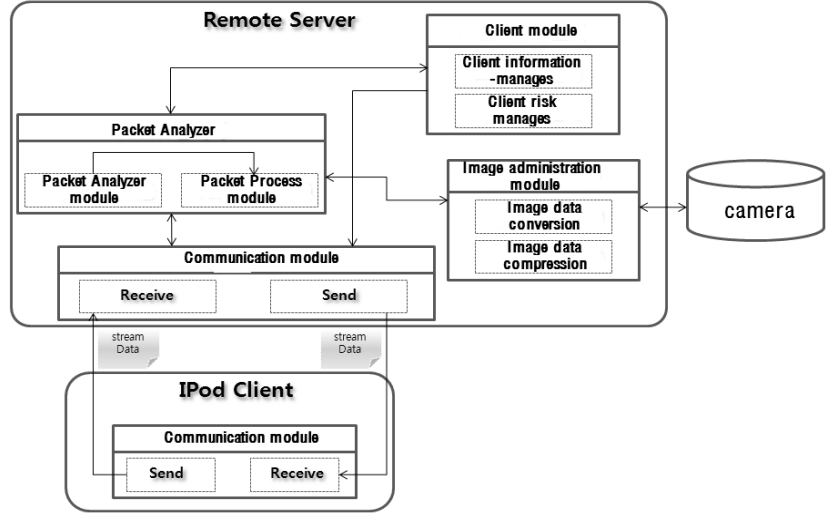

Fig. 6. The communication part from the remote server to iPhone client.

The iPod client module in Fig. 7 is composed of packet analyzer, motion management module, and image module. Each module receives a data in terms of motion information, vehicle condition information, and image data of transmitted data from server. In iPod client, data is transmitted following the movement of terminal for direction control of remote vehicle through motion module and it leads to perform the role which remote controls vehicle by server.

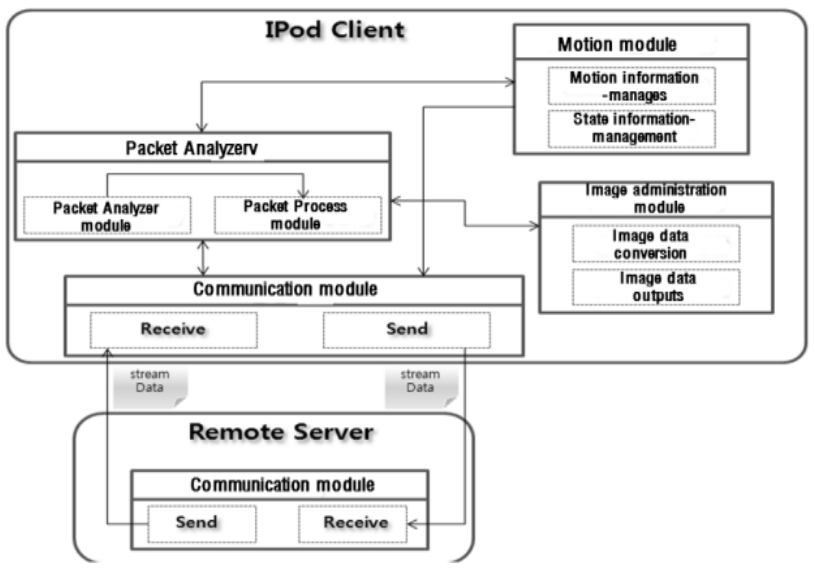

Fig. 7. The communication part from iPhone client to server PC

From the car client which is shown in Fig. 8, the role transmitting data to remote server by handling vehicle movement, condition information as well as image information is taken in charge. Especially, the transmitted information of motion control from iPod terminal is received via remote server and a basic signal for vehicle control is extracted by packet analyzer to control the direction of remote vehicle. 


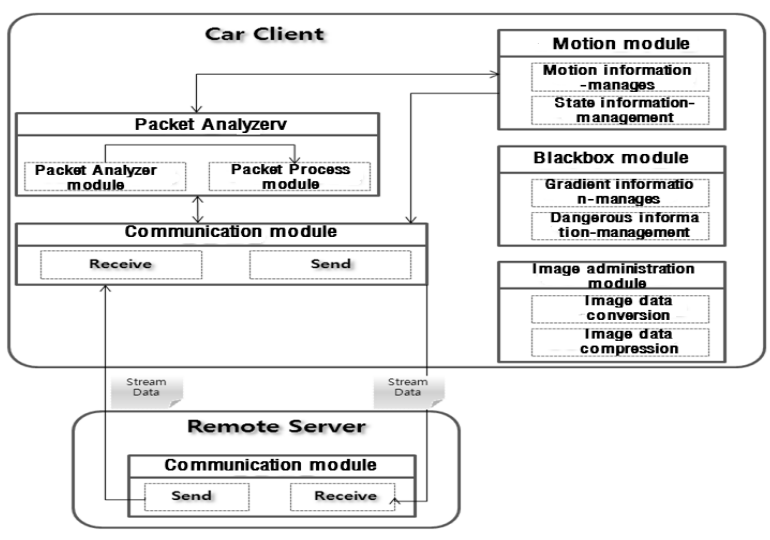

Fig. 8. The structure of remote controlled vehicle module.

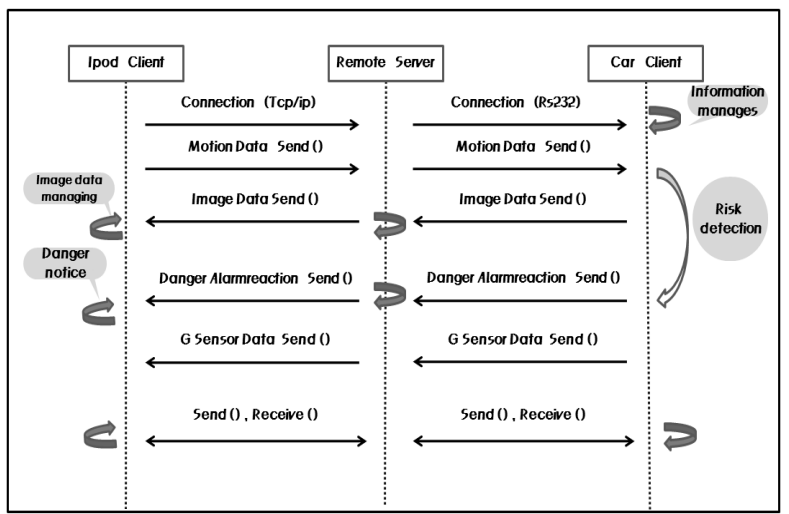

Fig. 9. Flow chart of vehicle remote control system.

Fig. 9 indicates the flow of communication and control of the whole system. iPod Client, first of all, becomes accessed with the use of remote server and $\mathrm{Wi}-\mathrm{Fi}$ or TCP/IP and vehicle control motion data is sent towards server to transmit data by separating into 5 stages as left, right, front, back, and stop of vehicle.

From Remote Server, communication is operated in accordance with vehicle and Bluetooth method and the motion data received from iPod terminal is transmitted to the car. In remote vehicle, the condition of vehicle can be verified by delivering the front obstacle information data to iPod terminal via remote sever through the image, scope condition, and ultrasonic sensor of vehicle.

Car Client transmits the vehicle condition and image data to remote server in real time to present the movement condition of vehicle with image. Moreover, it delivers the vehicle information to server in real time; thus, vehicle can be remote controlled in iPod terminal.

\section{VEHICLE REMOTE CONTROL EQUIPMENT AND BLACK BOX}

The part of vehicle remote control is controlled by using the 3-axis sensor of G-sensor installed from iPhone/iPod touch.

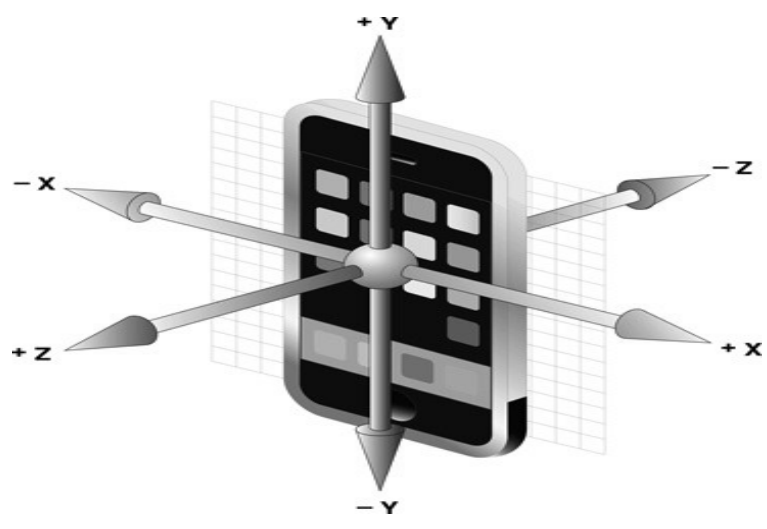

Fig. 10. The iPhone has G sensor with 3-axis.

G-sensor notices the inertial force working on a particular direction to measure the acceleration and gravity. As Fig. 10 shows, the size of acceleration can be measured with 3-axis of $\mathrm{X}, \mathrm{Y}, \mathrm{Z}$ direction.

Instead of vehicle steering equipment handle, this terminal G-sensor is used to remote control vehicle and the changed value of axis is transmitted to the server as a data, since Wi-Fi communication is available for the terminal instantly. The server received data is sent over the vehicle through Bluetooth communication module and DC motor which receives this data value is being controlled in direction of front and back; besides that, AC motor is driven by controlling in terminal in the left and right direction. G-sensor not only controls the direction, but it also can control the velocity following the movement of axis [4].

Fig. 11, as a method to control vehicle with direction control algorithm, is to move according to each scope into $\mathrm{X}, \mathrm{Y}, \mathrm{Z}$ axis. Control value is operated to be saved directly to the auto-control algorithm following the motor reaction of RC car which is utilized in this study. As a result, left and right direction operate the scope to $\mathrm{X}$-axis from -0.5 to 0.5 ; the front and back operate by using scope between $-0.8 \sim 0.8$ with 0 as the center of $Z$-axis; and stop is not operated when the value of $\mathrm{Y}$-axis is 0 . Also, the velocity control of vehicle can control the velocity with several stages of vehicle control.

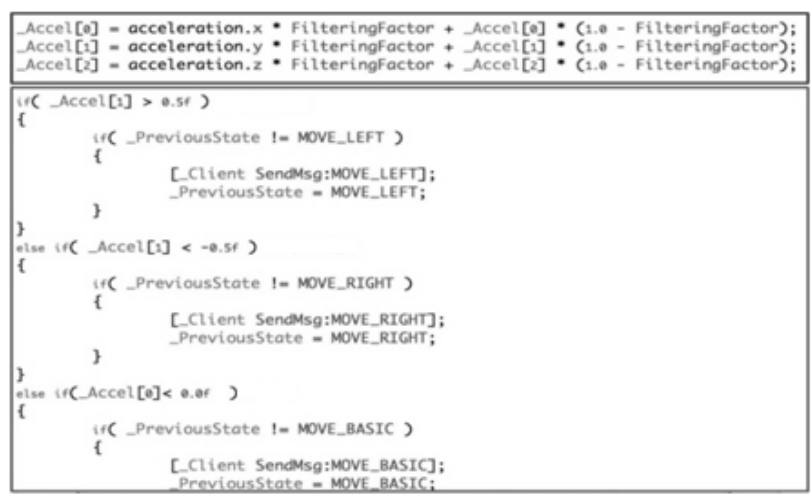

Fig. 11. Directional control algorithm. 
Car black box system utilizes scope sensor and ultrasonic sensor camera to identify the condition of vehicle. The vehicle condition about tow or tire problem can be identified with receiving signal from iPhone terminal by loading scope sensor at the center of vehicle to send data to server following the designated scope value of vehicle. If vehicle and driver are departed when there is car accident or the back side is impacted, ultrasonic sensor can identify the image transmission in real time and save through server and Smart Phone with push notification service as shown in Fig. 12.

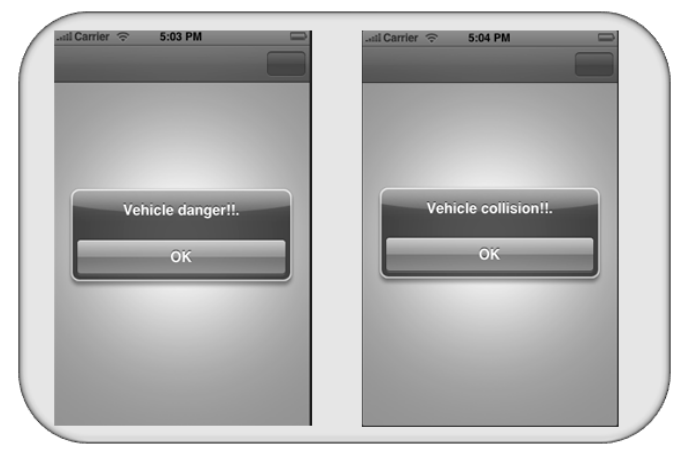

Fig. 12. The vehicle status information.

\section{EXPERIMENT AND RESULT}

\section{A. Experiment environment}

Experimental environment is shown in Table I. Vehicle model to detect some sensing information should be used and iPhone is used to control its speed and direction of the vehicle model. Control algorithms through the use of mobile devices according to the movement speed and direction can be applied.

TABLE I

DEVELOPMENT ENVIRONMENTS

\begin{tabular}{|c|c|c|}
\hline & Server & iPhone \\
\hline Development & Windows XP & Mac OS \\
\hline System Tool & MS MFC library & X-code \\
\hline $\begin{array}{c}\text { Communication } \\
\text { method }\end{array}$ & $\begin{array}{c}\text { Wi-Fi(802.11b), } \\
\text { Bluetooth }\end{array}$ & $\begin{array}{c}\text { Wi-Fi } \\
(802.11 \mathrm{~b})\end{array}$ \\
\hline Image method & Open CV & Open GL \\
\hline
\end{tabular}

According to the performance of $\mathrm{DC}$ and $\mathrm{AC}$ motor, left and right control is appointed by the $\mathrm{X}$ axis -0.5 to 05 , and front and rear speed is appointed by the Z-axis -0.5 to 0.5 . It shows the experimental results of limited control of the vehicle, and this part can be controlled at any time freely by the status of the motor.

\section{B. Examination Result}

Fig. 13, 14, and 15 are the part of remote control, vehicle communication, and terminal among the realized system. By using this realized system, $d s$ mobile (Open access point of Dongseo University) $802.11 \mathrm{~b} / \mathrm{g}$ wireless adapter is utilized to examine in the university where is available for Wi-Fi.

As a result, the image data is sent over server from the vehicle and being monitored; moreover, the image transmitted to server is sent over iPhone terminal in real time. The result also indicates that vehicle directional control and vehicle status information, except image, are operated in everywhere in Wi-Fi area of the school without any errors [5].

Experimental environment is split into two parts. First, PC servers are used typically in the MFC C-language on Windows XP OS environment to the server environment, implemented through the Open CV library image and direction between the vehicle and the server to control data communications Bluetooth RS232c is transmitted through the method.

The Bluetooth data communication profit is limited in the mobile terminal running the program in the MAC OS through X-code program in the terminal and the mobile terminal iPhone uses Wi-Fi and elementarily transmits the vehicle control data.

In addition, by using the OpenGL library unlike the server platform, the image screen seen as a mobile shows the image video to a screen.

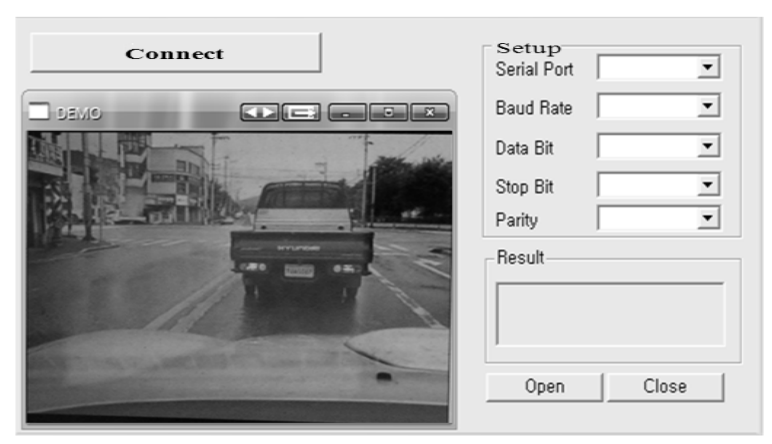

Fig. 13. Remote server communication connectivity and image.

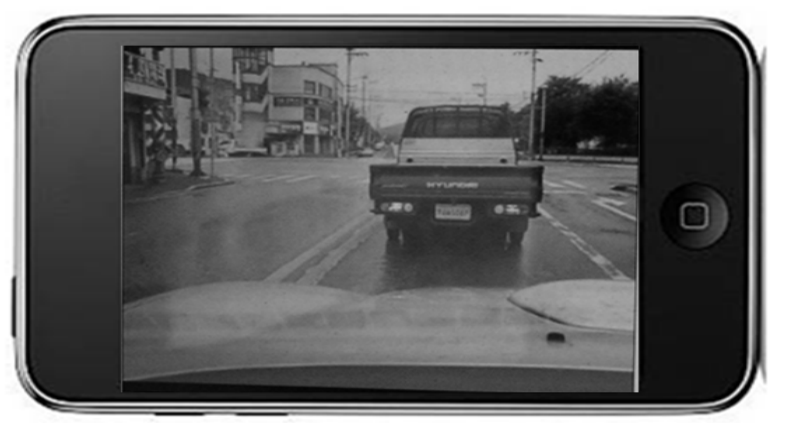

Fig. 14. Terminal image of vehicle control. 


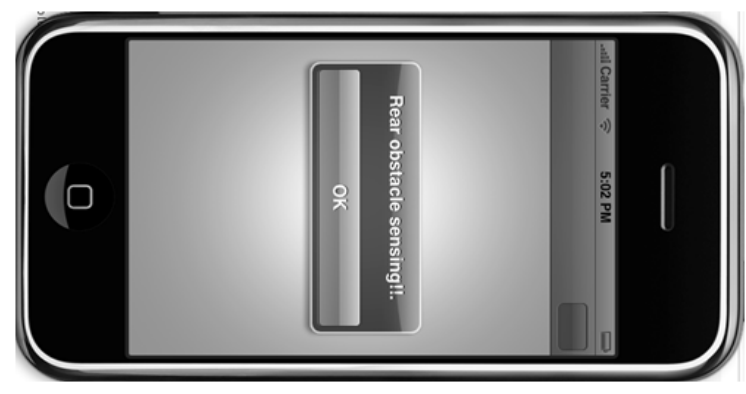

Fig. 15. Vehicle status of terminal notification signal.

\section{CONCLUDING REMARKS}

This paper has suggested and designated the way to control vehicle with Wi-Fi or bluetooth communication by using iPhone mounted G-sensor. From the experiment results, the vehicle model can be controlled by iPhone with wireless, although there is some limitation for communication distance between the control terminal and moving vehicle.

However, Wi-Fi service is accomplished in the part of capital area until now; thus, it is simple limitation for examination circumstance. This means that such environmental restriction for remote control is still remained, because the service is unstable even in the service available area. But the user is capable to control the car in $3 \mathrm{G}$ network in outdoor without Wi-Fi access point.

The result of this study has a great significance that is a starting point of vehicle remote control development by using Smart Phone like iPhone. If wireless LAN and WiBro is more comfortably available to access the network, various controlling vehicle by using a further portable terminal will be possible.

Furthermore, the control system developed in this study can be applied to the automation of distribution management system; bidirectional sensor network environment structure will be available with using various sensors; and several changes will be brought through applying from home network [6].

\section{ACKNOWLEDGMENT}

This work was supported in part by the Dongseo Frontier 2009 and the research foundation of Dongseo University in 2010.

\section{REFERENCES}

[1] Mun Byeong-Hyeon, Jin Yeong-Suk, Ryu Jeong-Tak "the sensor for vehicle system implementation using the radio communication and mobile apparatus", and Korea industrial information institute dissertation.

[2] Kim Gyeong-geun, Jung Won-Su, O Yeong-Hwan "the remote control embedded system using the wireless LAN", Korea institute of communication sciences article the 33 volume number 4(communication industry application), pp.105-112, and 2008.

[3] Jee Ui-gyeong, Sung Jun-Yong, Han Min-Hong "the vehicle radio control using WiBro", Korea SAE estimation thesis collection of scientific meeting, pp.1550-1554, and 2006.

[4] Lee Ju-Heon, Lee Hyeon-Seung, Song Ha-Yun, Park Jun "the embedded software design for the mobile sensor vehicle", Korea information science society collection of learned papers for fall academic presentation the 34 volume number 2 (B), pp.417-422, and 2007.

[5] Pak Jeong-Hyeon, Lee Min-Yeung, Sim Gwi-Bo "the mobile robot and network camera base intelligent monitoring system design", and Korea intelligence data acquisition control system institute.

[6] Park Jae-Hong, Son Yeong-Jin, Kim Jeong-Ha "the improved unmanned vehicle remote control system design", Korea SAE electrical \& electronics, ITS section symposium, pp.113-119, 2005 .

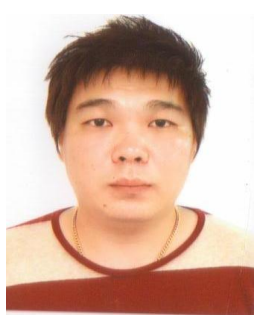

Jong Gun Song Received B.S degree in Network Information Engineering, Dongseo University in 2009 . He will be received M.S degree of Ubiquitous IT, Dongseo University in February 17, 2011. Field of study is Smart Phone, Remote Control, Vehicle, and Wi-Fi application areas.

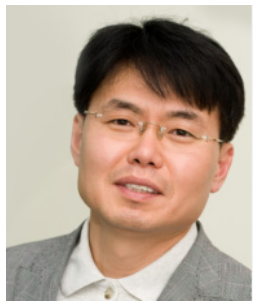

Won-Tae Jang received BS Degree in electronic engineering from SungKyunKwan University, Seoul, Korea in 1985, and received MS Degree in electronic engineering from University of Seoul, Korea in 1995. He is currently an assistant professor in the School of Computer and Information Engineering at Dongseo University. From 1989 to 2000, he was a research associate at the Korea Telecom International. His current research interests include developing mobile communication system, RFID system.

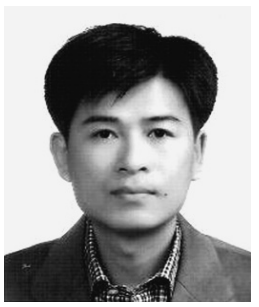

Tae Yong Kim was born in Korea, on November 14, 1965. He received a Ph.D. at Okayama University, Graduate School of Natural Science and Technology, in 2001 and is presently an assistant professor at Dongseo University, Korea. He was worked on analysis of antenna device, flow sensor system, numerical modeling, and simulation for various fields. Member of Institute of Control, Robotics and System and the Korea Institute of Maritime Information \& Communication Sciences. 